\title{
GELATIN AS A PLASMA SUBSTITUTE: THE EFFECTS OF INTRAVENOUS \\ INFUSION OF GELATIN ON CARDIAC OUTPUT AND OTHER ASPECTS \\ OF THE CIRCULATION OF NORMAL PERSONS, OF CHRONICALLY ILL PATIENTS, AND OF NORMAL VOLUNTEERS SUBJECTED TO LARGE HEMORRHAGE
}

\author{
By A. G. FLETCHER, JR., JAMES D. HARDY, C. RIEGEL, AND C. E. KOOP \\ (From the Harrison Department of Surgical Research, School of Medicine, University of \\ Pennsylvania; the Medical Clinic of the Hospital of the University of Pennsylvania; \\ and the Research Department of Therapeutics, University of \\ Pennsylvania, Philadelphia)
}

(Received for publication June 19, 1944)

Recent experimental and clinical studies have demonstrated that especially prepared ossein gelatin, administered intravenously, is a safe and effective plasma substitute for use in the treatment of shock and is useful in certain hypoproteinemic states in man (1 to 3 ). Other investigators have suggested that gelatin shows some promise as a source of nutritional protein for intravenous administration $(4,5)$. It has seemed important to us that these studies should be extended to include observations on the dynamics of the circulation as affected by the intravenous infusion of the gelatin colloid in man.

There are available a number of reports on the circulatory effects of the intravenous infusion of saline or glucose solutions (6 to 11) and of colloidal solutions such as whole blood, plasma, or acacia (12 to 15 ). We have observed the changes in pulse rate, blood pressure, blood volume, venous pressure, cardiac output, hematocrit, and plasma protein produced by the intravenous infusion of gelatin in normal subjects, chronically ill patients, and volunteers subjected to experimental hemorrhage. The effect of hemorrhage per se proved to be of particular interest and has been the subject of a separate paper prepared in collaboration with others who were concurrently engaged in the study of this problem $(16,17)$.

Of the work reported here, the clinical studies carried out on normal persons, on patients, and on the volunteers after hemorrhage, recorded in Tables II, III, and IV, were made by Doctors Fletcher and Hardy. Unfortunately, these authors were inducted into the armed forces before the study was quite completed. The chemical analyses were all performed by Dr. Riegel. The estimations of blood volume were all made by $\mathrm{Dr}$.
Koop. The special study on venous pressure after gelatin infusion was carried out by Dr. Koop with the kind assistance of Dr. R. L. Mayock. We are indebted to Dr. Isaac Starr for assistance in the final preparation of the manuscript.

\section{MATERIAL AND METHODS}

The gelatin used in this study was specially prepared for intravenous use by the hydrolysis of alkali-treated long bone collagen under carefully standardized conditions. ${ }^{1}$ The material was made up in a 6 per cent solution in 0.9 per cent saline and autoclaved in $500 \mathrm{ml}$. containers at 15 pounds pressure for 20 minutes.

It should be pointed out that several different preparations of gelatin are now under investigation. Since they vary in respect to size of gelatin molecules and colloid osmotic pressure, and, therefore, in their physiological effects, the data presented herewith are to be considered only in relation to the specific preparation described. The gelatin solution used in these studies has been designated P-20.

The normal subjects studied were healthy male volunteers between the ages of 18 and 50 years. A group of 5 chronically ill patients, ranging in age from 14 to 65 , were also selected for a study from the wards of the University Hospital.

In the hemorrhage experiments, venous blood was drawn under mild suction through a large bore needle into a graduated collecting bottle.

Estimates of the cardiac output were made by the use of the ballistocardiograph, the results being calculated according to the area method (18). The results are reported as percentage deviation from an empirical average normal value, the normal limits being \pm 22 per cent. These studies were carried out in the laboratory of the Hartzell Research Department of Therapeutics through the courtesy of Dr. Isaac Starr.

Specimens of venous blood were drawn with a minimum of stasis at suitable intervals for the determination

\footnotetext{
1 Prepared and supplied by Dr. D. Tourtellotte, Charles
} B. Knox Gelatin Company, Johnstown, N. Y. 
of the hematocrit and the concentration of plasma protein and gelatin. Hematocrit determinations were made in duplicate on oxalated blood in Van Allen tubes centrifuged at 2500 r.p.m. for 30 minutes. Analyses for plasma protein and gelatin were carried out by differential precipitation and nitrogen determination by a Kjeldahl semimicro technic.

Estimates of the blood volume were made from the dilution of the dye T1824 by a technic modified from that of Gibson and Evans (19).

Venous pressure was determined by an adaptation of the direct method of Moritz and Tabora (20) using a manometer filled with citrate solution and connected by a 3-way stop-cock to the infusion apparatus. Subjects lay supine, and the zero point selected was one-half of the depth of the chest, measured from the angle of Louis to the surface of the bed. No reading was accepted unless the respiratory variations could be clearly seen.

For some time, a single needle inserted in a vein was used both to measure venous pressure and to introduce the gelatin, and this is the technic usually adopted. Our experiments had been nearly completed before the hazard of this procedure was realized.

In every estimate of venous pressure by the technic of filling a burette and allowing the fluid to run into the vein as far as it will, the final reading should be corrected for capillarity and resistance in the tubing and needle. Thus, when the needle of our venous pressure apparatus is immersed in a beaker of water and the stopcock opened, the column in the burette always comes to rest above the water level in the beaker. The amount of this difference, usually about $1 \mathrm{~cm}$., was subtracted from the burette readings to estimate venous pressure. Eventually we realized that the viscous gelatin by sticking in the needle might reduce its lumen and so increase the magnitude of this correction for resistance. This might, perhaps, account for the apparent increase in venous pressure found after gelatin infusions by ourselves and others. Therefore, experiments were designed to test the matter.

TABLE I

Estimation of venous pressure in arm veins before and after gelatin infusion, using the needle through which gelatin had been injected and also a clean needle of similar size

\begin{tabular}{|c|c|c|c|c|c|c|c|c|}
\hline \multirow{4}{*}{$\begin{array}{c}\mathrm{Pa}^{-} \\
\text {tient }\end{array}$} & \multicolumn{3}{|c|}{ Before gelatin infusion } & \multicolumn{5}{|c|}{ After gelatin infusion } \\
\hline & \multirow{3}{*}{$\begin{array}{c}\text { Blood } \\
\text { vol- } \\
\text { ume }\end{array}$} & \multirow{2}{*}{\multicolumn{2}{|c|}{$\begin{array}{l}\text { Venous } \\
\text { pressure }\end{array}$}} & \multirow{3}{*}{$\begin{array}{l}\text { Blood } \\
\text { vol- } \\
\text { ume }\end{array}$} & \multicolumn{4}{|c|}{ Venous pressure } \\
\hline & & & & & \multicolumn{2}{|c|}{$\begin{array}{l}\text { "Gelatin" } \\
\text { needle }\end{array}$} & \multicolumn{2}{|c|}{$\begin{array}{c}\text { "Clean" } \\
\text { needle }\end{array}$} \\
\hline & & $\underset{\text { arm }}{\text { Right }}$ & $\begin{array}{l}\text { Left } \\
\text { arm }\end{array}$ & & $\underset{\text { arm }}{\text { Right }}$ & $\begin{array}{l}\text { Left } \\
\text { arm }\end{array}$ & $\underset{\text { arm }}{\text { Right }}$ & $\begin{array}{l}\text { Left } \\
\text { arm }\end{array}$ \\
\hline & $m l$. & $\underset{H=0}{c m}$ & $\underset{H \mathbf{O}}{\mathrm{cm}}$ & $m l$. & $\stackrel{c m .}{H .0}$ & ${ }_{H=0}^{c m}$. & $\underset{H \geqq 0}{c m}$ & $\underset{H=0}{c m}$. \\
\hline $\begin{array}{l}\text { E. M. } \\
\text { L. A. } \\
\text { T. O. }\end{array}$ & $\begin{array}{l}2638 \\
2638\end{array}$ & $\begin{array}{r}10.3 \\
6.5 \\
3.2\end{array}$ & $\begin{array}{l}7.6 \\
3.7 \\
6.4\end{array}$ & $\begin{array}{l}3040 \\
3590\end{array}$ & $\begin{array}{l}9.3 \\
8.1 \\
4.8\end{array}$ & $\begin{array}{l}8.1 \\
6.6 \\
7.9\end{array}$ & $\begin{array}{l}9.6 \\
6.9 \\
4.6\end{array}$ & $\begin{array}{l}9.0 \\
7.0 \\
7.9\end{array}$ \\
\hline
\end{tabular}

It was easy to demonstrate that such an error might occur. The apparatus used for venous pressure was employed, the 20 gauge needle being immersed in a beaker of water at room temperature $\left(24^{\circ}\right.$ C. $)$. A series of readings showed that the burette column came to rest 0.9 , $0.9,0.8,0.8$, and $0.9 \mathrm{~cm}$. above the fluid level in the beaker. Then, mimicking the technic of intravenous injection, $250 \mathrm{ml}$. of gelatin was passed through the needle. After this the column in the burette came to rest 3.5, 3.2, 2.9, $3.2,3.0$, and $3.0 \mathrm{~cm}$. above the water level in the beaker. Cleaning the needle restored the original resistance. The failure to correct for such a change in resistance would account for the increased value for venous pressure after gelatin, recorded in several, but by no means all, of our experiments.

However, the difference in resistance caused by gelatin in the example cited was the largest we encountered in many similar experiments with 20 or 19 gauge needles, the size we employed for venipuncture. Furthermore, if the needles were kept at body temperature by immersion in water at $37^{\circ} \mathrm{C}$., perfusing gelatin through them had no noteworthy effect on the resistance of the system. Also, the results obtained at room temperature were most irregular and increases in resistance after gelatin varied from 0 to $2.2 \mathrm{~cm}$. Obviously, the result depends on the many factors affecting the viscosity which varies with the type of gelatin used, the temperature, the degree of dilution with saline used to fill the tubing, and especially with the cleanliness of the needle.

To study the matter further, venous pressures were measured in both arms of 3 patients, before and after infusions of gelatin. After the infusion, the estimate was made by using the needle through which the gelatin had been administered and also a second clean needle of similar gauge. The results, Table I, emphasize the variability of pressures measured in different peripheral veins. However, the averages showed that errors which could be attributed to clogging the needle with gelatin were negligible, so we accepted our results made with the original technic.

\section{RESULTS}

\section{A. Intravenous infusions of gelatin in normal male subjects}

The effects of 5 intravenous infusions of large amounts of gelatin were observed in 3 healthy male subjects. The data are presented in Table II, and Figure 1 shows the averages of the results obtained.

The 2 subjects who received gelatin twice experienced on each occasion a slight feeling of tightness in the chest during the infusion and also a throbbing headache which was most marked 3 or 4 hours after the infusion had been completed. This gradually disappeared during the subsequent 


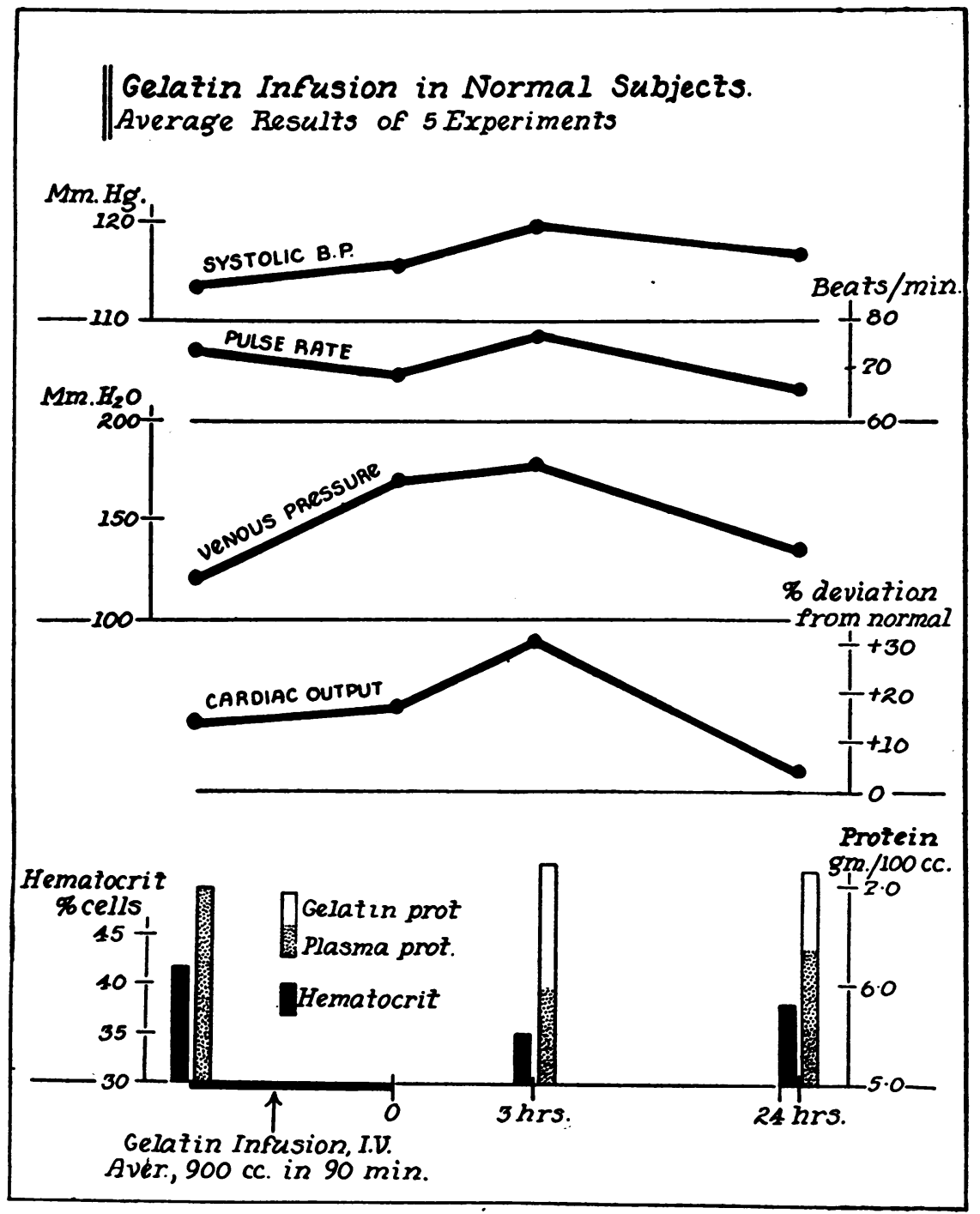

Fig. 1. Average Results Obtained in 5 Normal Subjects Before and for 24 Hours after a Large Infusion of Gelatin Intravenously

24 hours. There were no other untoward symptoms of any kind.

The results indicate that the pulse rate and blood pressure of healthy persons were little changed by a gelatin infusion. The average venous pressure was slightly increased by the infusion. The average cardiac output was not increased significantly immediately after the infusion but it became so 3 hours later, being well above normal limits at that time. This increase was not found 24 hours later. The fact that the last cardiac output was below the initial estimate is not to be attributed to the gelatin, for apprehension increases the cardiac output before most experimental procedures, and this passes off as the conclusion approaches.

Hematocrit and plasma protein concentration diminished markedly after the infusion. Indeed, the plasma proteins never, and the hematocrit only once, regained their initial values in 24 hours.

\section{B. Intravenous infusion of gelatin in chronically ill patients}

Somewhat less complete studies of the circulation were made during 6 infusions in 5 chronically ill patients of the type to whom gelatin might be 
given therapeutically for its nutritional value. The data are presented in Table III.

The outstanding feature in these cases was the striking increase in cardiac output, which was considerably greater than that observed in normal subjects receiving gelatin. The initial hyperkinemia, averaging +39 per cent above average normal, may be explained by the anemia and emaciation of the patients, but excitement from the impending injection may well have been a factor also. That several of these patients had marked tachycardia before the injection is to be noted. In any event, the average cardiac output at the completion of the infusion had increased to +72 per cent above the average normal, and there was no febrile reaction nor, indeed, reaction of any kind to which the increase could be attributed.

\section{Intravenous infusion of gelatin following acute experimental hemorrhage}

Twelve healthy male volunteers between the ages of 18 and 50 were subjected to acute hemorrhage of measured amount. They were bled ap- proximately $15 \mathrm{ml}$. per kilogram of body weight, or between 16 and 20 per cent of the blood volume as estimated from their weight. Bleeding required from 8 to 20 minutes.

Nine subjects were observed for periods not exceeding 90 minutes after the bleeding. Each of these subjects received an intravenous infusion of gelatin amounting to approximately two-thirds of the volume of blood removed. The infusion was started about 15 minutes after bleeding had been completed. It required about 15 minutes to complete, and shortly thereafter the subjects were allowed to leave the laboratory. Detailed studies of the effect of acute hemorrhage in these and other cases have been reported elsewhere $(16,22$, 23). In summary, 5 out of 9 of our subjects exhibited a pronounced fall in blood pressure accompanied by pallor, sweating, bradycardia, and reduced cardiac output. Venous pressure was also reduced in most cases. There was a brief loss of consciousness in 1 subject. These episodes of circulatory collapse were of short duration, and the subjects usually showed rapid spontaneous improvement even before the infusion of

TABLE II

Intravenous infusion of gelatin in normal subjects

\begin{tabular}{|c|c|c|c|c|c|c|c|c|c|c|c|c|c|}
\hline \multirow{2}{*}{$\underset{\text { ject }}{\text { Sub- }}$} & \multicolumn{3}{|c|}{ Physical data } & \multirow{2}{*}{$\begin{array}{l}\text { Volume } \\
\text { of } \\
\text { gelatin } \\
\text { infused }\end{array}$} & \multirow{2}{*}{$\begin{array}{c}\text { Time } \\
\text { required } \\
\text { for } \\
\text { infusion }\end{array}$} & \multirow{2}{*}{$\begin{array}{c}\text { Time of } \\
\text { observation }\end{array}$} & \multirow{2}{*}{$\begin{array}{l}\text { Pulse } \\
\text { rate }\end{array}$} & \multirow{2}{*}{$\begin{array}{c}\text { Blood } \\
\text { pressure }\end{array}$} & \multirow{2}{*}{$\begin{array}{l}\text { Periph- } \\
\text { eral } \\
\text { ivenous } \\
\text { pressure }\end{array}$} & \multirow{2}{*}{$\begin{array}{c}\text { Cardiac } \\
\text { output, } \\
\text { deviation } \\
\text { from } \\
\text { average } \\
\text { normal }\end{array}$} & \multirow{2}{*}{$\begin{array}{l}\text { Plasma } \\
\text { protein }\end{array}$} & \multirow{2}{*}{$\begin{array}{l}\text { Plasma } \\
\text { gelatin }\end{array}$} & \multirow{2}{*}{$\underset{\text { tocrit }}{\text { Hema- }}$} \\
\hline & Weight & Height & $\begin{array}{l}\text { Sur- } \\
\text { face } \\
\text { area }\end{array}$ & & & & & & & & & & \\
\hline A. F. & $\begin{array}{c}\mathrm{kgm} . \\
77\end{array}$ & $\begin{array}{l}c m . \\
187\end{array}$ & $\begin{array}{l}\text { sq. } m . \\
2.03\end{array}$ & $\begin{array}{c}m l . \\
1000\end{array}$ & $\begin{array}{c}\min . \\
65\end{array}$ & $\begin{array}{l}\text { Initial } \\
\text { After infusion } \\
4 \text { hrs. later } \\
26 \text { hrs. later }\end{array}$ & $\begin{array}{l}88 \\
83 \\
83 \\
71\end{array}$ & $\begin{array}{l}m m . H g \\
118 / 70 \\
126 / 72 \\
124 / 66 \\
116 / 70\end{array}$ & $\begin{array}{c}m m . \mathrm{H}_{2} \mathrm{O} \\
98 \\
147\end{array}$ & $\begin{array}{l}\text { per cent } \\
+30 \\
+37 \\
+41 \\
+4\end{array}$ & $\begin{array}{c}\text { grams } \\
\text { per } \\
100 \mathrm{ml} . \\
7.33 \\
\\
6.91 \\
6.51\end{array}$ & $\begin{array}{c}\text { grams } \\
\text { per } \\
100 \mathrm{ml} .\end{array}$ & $\begin{array}{c}\text { percent- } \\
\text { age of } \\
\text { cells } \\
39.0 \\
\\
37.0 \\
39.0\end{array}$ \\
\hline J. H. & 73 & 180 & 1.92 & 1000 & 70 & $\begin{array}{l}\text { Initial } \\
\text { After infusion } \\
3 \mathrm{hrs} \text {. later } \\
26 \mathrm{hrs} \text {. later } \\
52 \mathrm{hrs} \text {. later }\end{array}$ & $\begin{array}{l}71 \\
72 \\
79 \\
66 \\
62\end{array}$ & $\begin{array}{l}110 / 76 \\
100 / 70 \\
120 / 80 \\
112 / 78 \\
114 / 74\end{array}$ & $\begin{array}{r}98 \\
148 \\
144 \\
112 \\
134\end{array}$ & $\begin{array}{r}+4 \\
0 \\
+35 \\
+13 \\
0\end{array}$ & $\begin{array}{l}7.53 \\
6.00 \\
6.44 \\
6.56\end{array}$ & $\begin{array}{l}1.39 \\
0.79 \\
0.75\end{array}$ & $\begin{array}{l}43.5 \\
34.5 \\
37.0 \\
36.0\end{array}$ \\
\hline J. W. & 84 & 185 & 2.09 & 1000 & 120 & $\begin{array}{l}\text { Initial } \\
\text { After infusion } \\
3 \text { hrs. later } \\
24 \text { hrs. later }\end{array}$ & $\begin{array}{l}72 \\
60 \\
64 \\
63\end{array}$ & $\begin{array}{l}118 / 66 \\
124 / 76 \\
120 / 66 \\
122 / 68\end{array}$ & $\begin{array}{l}140 \\
165 \\
178 \\
144\end{array}$ & $\begin{array}{r}+4 \\
0 \\
-\quad 2 \\
+\quad 9\end{array}$ & $\begin{array}{l}6.45 \\
\\
5.27 \\
5.99\end{array}$ & $\begin{array}{l}1.37 \\
1.03\end{array}$ & $\begin{array}{l}40.0 \\
35.0 \\
37.5\end{array}$ \\
\hline A. $\mathbf{F}$. & 84 & 187 & 2.09 & 790 & 90 & $\begin{array}{l}\text { Initial } \\
\text { After infusion } \\
4 \mathrm{hrs} \text {. later } \\
28 \mathrm{hrs} \text {. later }\end{array}$ & $\begin{array}{l}70 \\
63 \\
78 \\
62\end{array}$ & $\begin{array}{l}110 / 60 \\
116 / 68 \\
118 / 68 \\
114 / 70\end{array}$ & $\begin{array}{r}\mathbf{r} 125 \\
\mathbf{1} 197 \\
210 \\
1145\end{array}$ & $\begin{array}{l}+17 \\
+17 \\
+24 \\
-11\end{array}$ & $\begin{array}{l}6.49 \\
5.61 \\
6.44\end{array}$ & $\begin{array}{l}1.12 \\
0.56\end{array}$ & $\begin{array}{l}43.0 \\
32.5 \\
38.5\end{array}$ \\
\hline J. H. & 84 & 180 & 2.09 & 750 & 90 & $\begin{array}{l}\text { Initial } \\
\text { After infusion } \\
3 \text { hrs. later }\end{array}$ & $\begin{array}{l}70 \\
68 \\
81\end{array}$ & $\begin{array}{l}110 / 76 \\
108 / 64 \\
114 / 84\end{array}$ & $\begin{array}{l}110 \\
164\end{array}$ & $\begin{array}{l}+17 \\
+30 \\
+50\end{array}$ & $\begin{array}{l}6.88 \\
5.86\end{array}$ & 1.09 & $\begin{array}{l}42.0^{\circ} \\
36.0\end{array}$ \\
\hline
\end{tabular}


TABLE III

Intravenous infusion of gelatin in chronically ill patients

\begin{tabular}{|c|c|c|c|c|c|c|c|c|c|c|c|c|c|}
\hline \multirow{2}{*}{$\underset{\text { ject }}{\text { Sub- }}$} & \multicolumn{3}{|c|}{ Physical data } & \multirow{2}{*}{ Diagnosis } & \multirow{2}{*}{$\begin{array}{l}\text { Serum } \\
\text { protein }\end{array}$} & \multirow{2}{*}{$\begin{array}{l}\text { Hemo- } \\
\text { globin }\end{array}$} & \multirow{2}{*}{$\begin{array}{c}\text { Vol- } \\
\text { ume of } \\
\text { gelatin } \\
\text { infused }\end{array}$} & \multirow{2}{*}{$\begin{array}{l}\text { Time } \\
\text { re- } \\
\text { quired } \\
\text { for } \\
\text { in- } \\
\text { fusion }\end{array}$} & \multirow{2}{*}{$\begin{array}{c}\text { Time of } \\
\text { observation }\end{array}$} & \multirow{2}{*}{$\begin{array}{l}\text { Pulse } \\
\text { rate }\end{array}$} & \multirow{2}{*}{$\begin{array}{c}\text { Blood } \\
\text { pressure }\end{array}$} & \multirow{2}{*}{$\begin{array}{l}\text { Periph- } \\
\text { eral } \\
\text { venous } \\
\text { pressure }\end{array}$} & \multirow{2}{*}{$\begin{array}{l}\text { Cardiac } \\
\text { output, } \\
\text { deviation } \\
\text { from } \\
\text { average } \\
\text { normal }\end{array}$} \\
\hline & Weight & Height & Age & & & & & & & & & & \\
\hline L. R. & $\begin{array}{c}k g m . \\
44\end{array}$ & $\begin{array}{l}c m . \\
155\end{array}$ & $\begin{array}{l}\text { yrs. } \\
33\end{array}$ & Carcinomatosis & $\begin{array}{c}\text { grams } \\
\text { per } 100 \\
\text { ml. } \\
3.8\end{array}$ & $\begin{array}{c}\text { per cent } \\
64\end{array}$ & $\begin{array}{l}m l . \\
500\end{array}$ & $\begin{array}{l}\min . \\
60\end{array}$ & $\begin{array}{l}\text { Initial } \\
\text { After infusion }\end{array}$ & $\begin{array}{l}125 \\
122\end{array}$ & $\begin{array}{l}m m . H_{g} \\
120 / 74 \\
118 / 72\end{array}$ & $m m . H_{2} \mathrm{O}$ & $\begin{array}{l}\text { per cent } \\
+54 \\
+70\end{array}$ \\
\hline L. $\mathrm{R}$. & 44 & 155 & 33 & Carcinomatosis & 3.8 & 64 & 1000 & 150 & $\begin{array}{l}\text { Initial } \\
\text { After infusion }\end{array}$ & $\begin{array}{l}120 \\
127\end{array}$ & $\begin{array}{r}90 / 70 \\
110 / 74\end{array}$ & & $\begin{array}{r}+65 \\
+122\end{array}$ \\
\hline A. $\mathrm{S}$. & 49 & 165 & 65 & $\begin{array}{l}\text { Carcinomatosis } \\
\text { Mild peripheral } \\
\text { edema }\end{array}$ & 5.8 & 75 & 1000 & 120 & $\begin{array}{l}\text { Initial } \\
\text { After infusion }\end{array}$ & $\begin{array}{l}82 \\
85\end{array}$ & $\begin{array}{l}120 / 70 \\
126 / 74\end{array}$ & $\begin{array}{r}92 \\
164\end{array}$ & $\begin{array}{l}-4 \\
+26\end{array}$ \\
\hline J. S. & 56 & 167 & 34 & $\begin{array}{l}\text { Subdural hema- } \\
\text { toma } \\
\text { Increased intra- } \\
\text { cranial pressure }\end{array}$ & 6.9 & 90 & 1000 & 90 & $\begin{array}{l}\text { Initial } \\
\text { After infusion } \\
1 \text { hour later }\end{array}$ & $\begin{array}{l}72 \\
80 \\
71\end{array}$ & $\begin{array}{l}110 / 68 \\
130 / 70 \\
128 / 68\end{array}$ & $\begin{array}{r}95 \\
124 \\
162\end{array}$ & $\begin{array}{l}+17 \\
+37 \\
+39\end{array}$ \\
\hline G. B. & 44 & 165 & 44 & $\begin{array}{l}\text { Hodgkin's dis- } \\
\text { ease, advanced }\end{array}$ & 6.4 & 50 & 1000 & 90 & $\begin{array}{l}\text { Initial } \\
\text { After infusion } \\
3 \text { hours later }\end{array}$ & $\begin{array}{r}97 \\
102 \\
102\end{array}$ & $\begin{array}{r}88 / 58 \\
104 / 64 \\
98 / 64\end{array}$ & & $\begin{array}{r}+78 \\
+104 \\
+82\end{array}$ \\
\hline J. M. & 49 & 157 & 14 & $\begin{array}{l}\text { Subacute glomer- } \\
\text { ulonephritis } \\
\text { Moderate edema }\end{array}$ & 3.52 & 55 & 300 & 90 & $\begin{array}{l}\text { Initial } \\
\text { After infusion }\end{array}$ & $\begin{array}{r}98 \\
103\end{array}$ & $\begin{array}{l}122 / 88 \\
128 / 98\end{array}$ & & $\begin{array}{l}-9 \\
+2\end{array}$ \\
\hline
\end{tabular}

gelatin was begun. Nevertheless, the rapid infusion of gelatin solution was always followed by prompt and complete relief of symptoms, and blood pressure, venous pressure, and cardiac output were rapidly restored to values equal to, or somewhat above, those observed initially. A typical record is shown in Figure 2, and the observations are recorded in Table IV. It will be noted that the reduction of plasma protein and hematocrit was considerably greater than dilution by the volume of gelatin injected would account for.

In contrast to results obtained on volunteers bled a corresponding amount and not given replacement therapy (16), all of those receiving gelatin were able to stand and walk without dizziness or faintness at intervals as brief as 30 to 45 minutes after a hemorrhage of $1000 \mathrm{ml}$. They then went home, or continued their usual activities, and later reported no untoward symptoms of any sort, with the exception of a rather severe thrombosis of the injected vein in 1 case.

Three additional subjects were studied over a period of 24 hours following hemorrhage comparable to that described above. One subject
(J. H.) received no replacement therapy. The second (A. F.) received replacement with a volume of normal salt solution equal to the volume bled. The third (T. N.) received gelatin replacement equal to two-thirds of the volume bled. These subjects took no food for 6 hours before the experiment, but were allowed water as desired at all times and food as desired beginning 3 to 4 hours after the hemorrhage. The results obtained in J. H. have been recorded (16); but, as they make an interesting contrast with those secured in the 2 cases who received replacement therapy, they have been summarized in Figure 3 .

The effects of hemorrhage in these 3 cases were similar to those observed in the other 9. All 3 exhibited a fall in blood pressure, bradycardia, pallor, and sweating, accompanied by subjective symptoms of faintness and nausea. There was a brief loss of consciousness in one subject (T. N.).

The outstanding differences among these 3 cases are seen in Figure 3. Subject J. H., with no replacement, showed only gradual hemodilution at a rate which suggested that the blood volume was still considerably below the initial level 20 hours after hemorrhage. A. F., after a rapid 
TABLE IV

Intravenous infusion of gelatin after acute hemorrhage

\begin{tabular}{|c|c|c|c|c|c|c|c|c|c|c|c|c|c|c|}
\hline \multirow[b]{2}{*}{$\underset{\text { ject }}{\text { Sub- }}$} & \multicolumn{3}{|c|}{ Physical data } & \multicolumn{2}{|c|}{ Volume bled } & \multirow[b]{2}{*}{\begin{tabular}{|c|} 
Vol- \\
ume \\
6 per \\
cent \\
gelatin \\
injected
\end{tabular}} & \multirow[b]{2}{*}{$\begin{array}{c}\text { Time of } \\
\text { observations }\end{array}$} & \multirow[b]{2}{*}{$\begin{array}{l}\text { Pulse } \\
\text { rate }\end{array}$} & \multirow[b]{2}{*}{$\begin{array}{c}\text { Blood } \\
\text { pressure }\end{array}$} & \multirow[b]{2}{*}{$\begin{array}{c}\text { Periph- } \\
\text { eral } \\
\text { venous } \\
\text { pressure }\end{array}$} & \multirow[b]{2}{*}{$\begin{array}{c}\text { Cardiac } \\
\text { output, } \\
\text { deviation } \\
\text { from } \\
\text { average } \\
\text { normal }\end{array}$} & \multirow[b]{2}{*}{$\begin{array}{l}\text { Plasma } \\
\text { protein }\end{array}$} & \multirow[b]{2}{*}{$\begin{array}{l}\text { Plasma } \\
\text { gelatin }\end{array}$} & \multirow[b]{2}{*}{$\begin{array}{c}\text { Hema- } \\
\text { tocrit }\end{array}$} \\
\hline & Weight & Height & $\begin{array}{l}\text { Sur- } \\
\text { face } \\
\text { area }\end{array}$ & Amount & $\begin{array}{c}\text { Percent- } \\
\text { age of } \\
\text { blood } \\
\text { volume } \\
\text { esti- } \\
\text { mated } \\
\text { from wt. }\end{array}$ & & & & & & & & & \\
\hline T. K. & $\begin{array}{c}k g m . \\
84\end{array}$ & $\begin{array}{l}\mathrm{cm} . \\
175\end{array}$ & $\begin{array}{l}s q . m . \\
2.00\end{array}$ & $\begin{array}{c}m l . \\
1200\end{array}$ & $\begin{array}{c}\text { per cent } \\
20\end{array}$ & $\begin{array}{l}m l . \\
840\end{array}$ & $\begin{array}{l}\text { Initial } \\
\text { After hemorrhage } \\
\text { After gelatin }\end{array}$ & $\begin{array}{l}74 \\
92 \\
78 \\
\end{array}$ & $\begin{array}{c}m m . ~ H g \\
\\
96 / 70 \\
84 / 64 \\
96 / 66 \\
\end{array}$ & \begin{tabular}{|c|}
$m m . H$ \\
\end{tabular} & $\begin{array}{c}\text { per cent } \\
-4 \\
=9 \\
-9\end{array}$ & $\begin{array}{c}\text { grams } \\
\text { per } \\
100 \mathrm{ml} . \\
6.20 \\
5.92 \\
4.30 \\
\end{array}$ & $\begin{array}{c}\text { grams } \\
\text { per } \\
100 \mathrm{ml} . \\
\\
1.23\end{array}$ & $\begin{array}{c}\text { percent- } \\
\text { age of } \\
\text { cells } \\
42.0 \\
39.5 \\
28.5\end{array}$ \\
\hline J. R. & 82 & 185 & 2.06 & 1065 & 17 & 710 & $\begin{array}{l}\text { Initial } \\
\text { After hemorrhage } \\
\text { After gelatin }\end{array}$ & $\begin{array}{l}66 \\
69 \\
72\end{array}$ & $\begin{array}{l}128 / 68 \\
130 / 70 \\
130 / 70\end{array}$ & $\begin{array}{l}146 \\
146 \\
150 \\
\end{array}$ & $\begin{array}{l}+13 \\
\frac{13}{+26} \\
\end{array}$ & $\begin{array}{l}6.36 \\
5.33 \\
4.90 \\
\end{array}$ & 1.00 & $\begin{array}{l}42.0 \\
39.0 \\
33.5 \\
\end{array}$ \\
\hline C. $\mathbf{M}$. & 61 & 170 & 1.72 & 900 & 18 & 600 & $\begin{array}{l}\text { Initial } \\
\text { After hemorrhage } \\
\text { After gelatin }\end{array}$ & $\begin{array}{l}71 \\
53 \\
62\end{array}$ & $\begin{array}{r}120 / 78 \\
58 / 40 \\
110 / 60\end{array}$ & $\begin{array}{l}164 \\
124 \\
164\end{array}$ & $\begin{array}{l}+4 \\
-11 \\
+13\end{array}$ & $\begin{array}{l}6.98 \\
5.13\end{array}$ & 0.87 & $\begin{array}{l}36.0 \\
28.0\end{array}$ \\
\hline B. B. & 68 & 173 & 1.81 & 1020 & 19 & 680 & $\begin{array}{l}\text { Initial } \\
\text { After hemorrhage } \\
\text { After gelatin }\end{array}$ & $\begin{array}{l}77 \\
77 \\
69\end{array}$ & $\begin{array}{l}114 / 60 \\
100 / 60 \\
108 / 58\end{array}$ & & $\begin{array}{l}+65 \\
+30 \\
+43\end{array}$ & & & $\begin{array}{l}36.5 \\
35.0 \\
29.0\end{array}$ \\
\hline E. T.* & 75 & 170 & 1.86 & 1050 & 19 & 750 & $\begin{array}{l}\text { Initial } \\
\text { After hemorrhage } \\
\text { After gelatin }\end{array}$ & $\begin{array}{l}89 \\
45 \\
76\end{array}$ & $\begin{array}{l}130 / 80 \\
120 / 70\end{array}$ & & $\begin{array}{l}+22 \\
-39 \\
-2\end{array}$ & & & $\begin{array}{l}48.5 \\
45.5 \\
37.0 \\
\end{array}$ \\
\hline H. G. & 69 & 173 & 1.81 & 1000 & 19 & 710 & $\begin{array}{l}\text { Initial } \\
\text { After hemorrhage } \\
\text { After gelatin }\end{array}$ & $\begin{array}{l}71 \\
58 \\
66\end{array}$ & $\begin{array}{r}118 / 70 \\
68 / 34 \\
108 / 60\end{array}$ & $\begin{array}{r}140 \\
95 \\
146\end{array}$ & $\begin{array}{r}+17 \\
0 \\
+13\end{array}$ & & & $\begin{array}{l}43.0 \\
39.0 \\
33.0\end{array}$ \\
\hline R. C. & 89 & 173 & 2.03 & 1020 & 17 & 680 & $\begin{array}{l}\text { Initial } \\
\text { After hemorrhage } \\
\text { After gelatin }\end{array}$ & $\begin{array}{l}67 \\
71 \\
77\end{array}$ & $\begin{array}{l}120 / 60 \\
120 / 80 \\
120 / 60\end{array}$ & $\begin{array}{l}140 \\
120\end{array}$ & $\begin{array}{l}-17 \\
-13 \\
+13\end{array}$ & & & $\begin{array}{l}39.5 \\
37.0 \\
29.5\end{array}$ \\
\hline L. J. & 73 & 180 & 1.92 & 1090 & 19 & 730 & $\begin{array}{l}\text { Initial } \\
\text { After hemorrhage } \\
\text { After gelatin } \\
\end{array}$ & $\begin{array}{l}112 \\
111 \\
94\end{array}$ & $\begin{array}{l}150 / 80 \\
130 / 80 \\
134 / 66\end{array}$ & $\begin{array}{l}170 \\
140 \\
175\end{array}$ & $\begin{array}{l}+48 \\
+48 \\
+35\end{array}$ & & & $\begin{array}{l}40.0 \\
38.0 \\
30.5\end{array}$ \\
\hline w. C.t & 76 & 178 & 1.91 & 920 & 16 & 760 & $\begin{array}{l}\text { Initial } \\
\text { After hemorrhage } \\
\text { After gelatin }\end{array}$ & $\begin{array}{l}74 \\
47 \\
78\end{array}$ & $\begin{array}{r}130 / 80 \\
70 / 40 \\
130 / 70\end{array}$ & & $\begin{array}{l}+17 \\
-17 \\
+13\end{array}$ & & & $\begin{array}{l}41.0 \\
39.5 \\
30.0\end{array}$ \\
\hline
\end{tabular}

* Standing B.P. 108/80.

† Standing B.P. 114/78.

hemodilution due to the saline infusion, showed no further hemodilution and, in fact, some hemoconcentration during the next 12 hours. Subject T. N., receiving gelatin replacement, showed the greatest reduction of hematocrit and plasma protein. The latter value found in the estimation made 6 hours after the start, is so low that we suspect an error although we were unable to find it.

After the hemorrhage, as long as the 3 subjects lay recumbent they were free of symptoms and differences in their pulse rate, blood pressure, and cardiac output were not conspicuous; but, as soon as the subjects arose, the differences were very conspicuous. The subject who had received gelatin was able to stand and walk without symptoms on his first attempt, 3 hours after hemorrhage, and had no trouble thereafter. In contrast, dizziness and a marked fall in blood pressure on arising persisted in the other 2 subjects for at least 12 hours, although in A. F., these symptoms were improved temporarily after the saline infusion.

After the rest of the experiments had been completed, to assist in the interpretation of the changes in hematocrit and plasma protein values found after gelatin, one of the authors (Koop) made estimates of blood volume before and after gelatin injection. Patient P. O. was chosen because he was judged to be well hydrated, having received a total of over $3000 \mathrm{ml}$. of fluid daily for 4 days, part by intravenous administration and the remainder through a gastrostomy. Patient J. O. was chosen because he was obviously dehydrated, as the only fluid he had received in the last 36 hours was $500 \mathrm{ml}$. of tap water by rectum. During this period, he had sweated profusely at operation, and at the time of the test he was extremely 


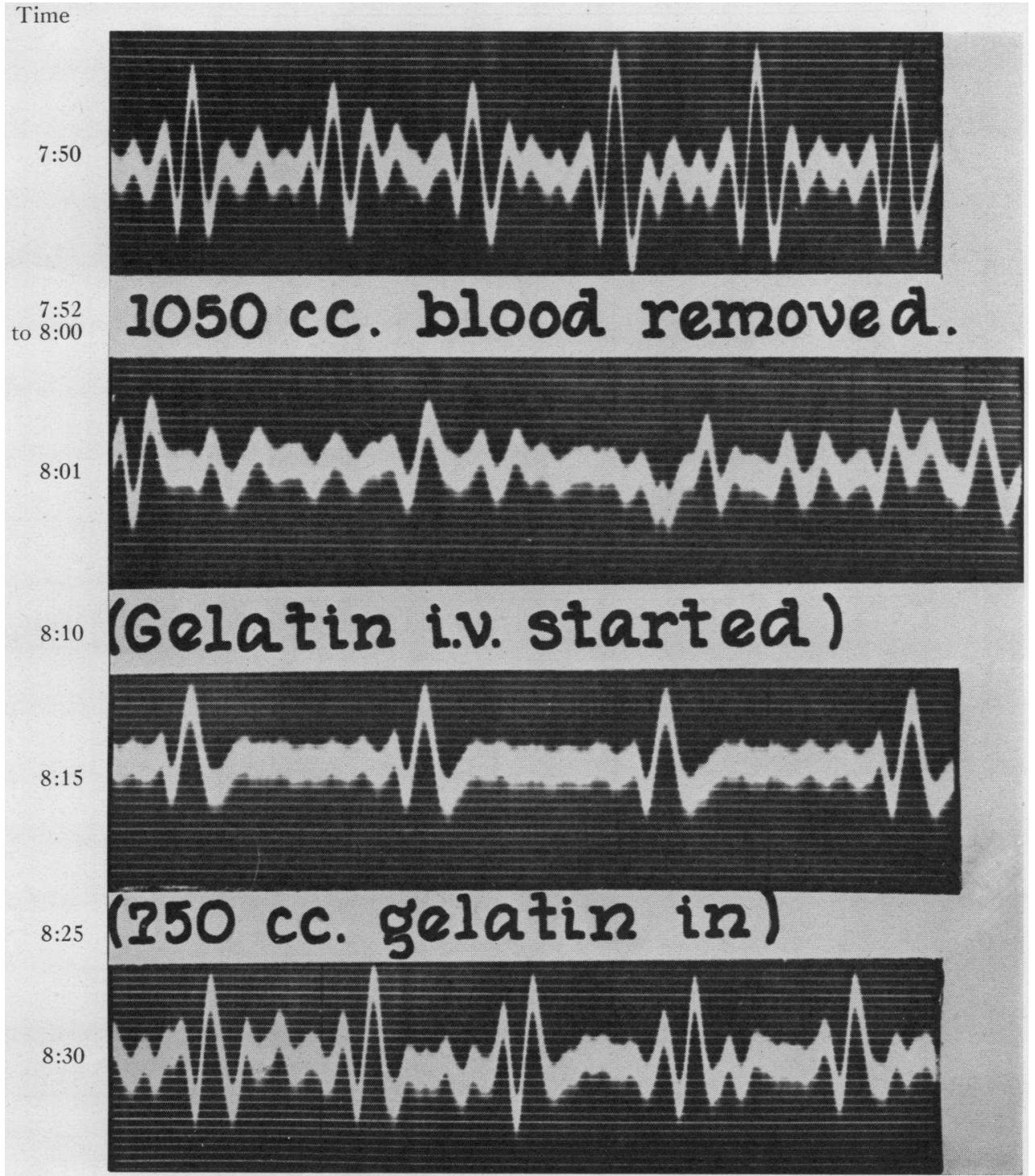

Fig. 2. Ballistocardiograms Before and After a Large Hemorrhage

The reproduction is actual size. Subject E. T., age 38, 5 feet 7 inches, 165 pounds.

At 7.50 , the B. P. $130 / 80$, the pulse rate 89 , the cardiac output +22 per cent above average normal (normal limits \pm 22 per cent).

After the hemorrhage, at 8.01 , the subject was sweating, pale, restless, and manifested greatly increased respiration. He complained that his vision was dim. Blood pressure could not be obtained. The pulse rate was 45 . The ballistocardiogram is distorted by the subject's restless movements and by impacts from respiratory movements. Selecting the undistorted complexes the cardiac output was calculated to be -40 per cent.

At 8.15 , after gelatin had been started, the symptoms were much improved, B. P. 50/30, pulse 52 , cardiac output -30 per cent.

At 8.30 , after completion of the infusion, the subject had no symptoms, the B. P. $110 / 72$, the pulse rate 77 , the cardiac output did not deviate from average normal. 


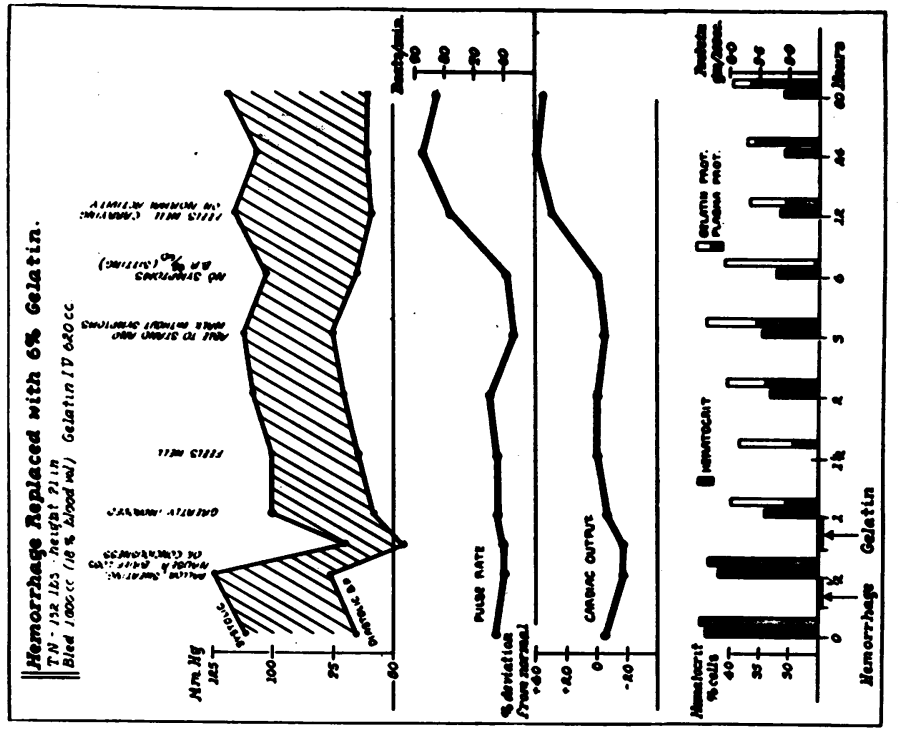

䓵

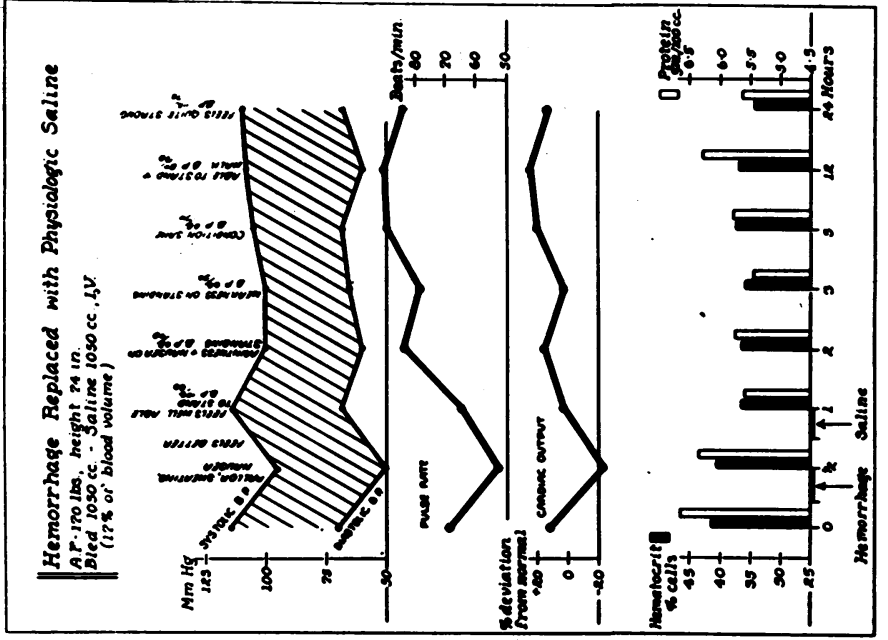

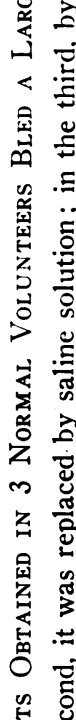

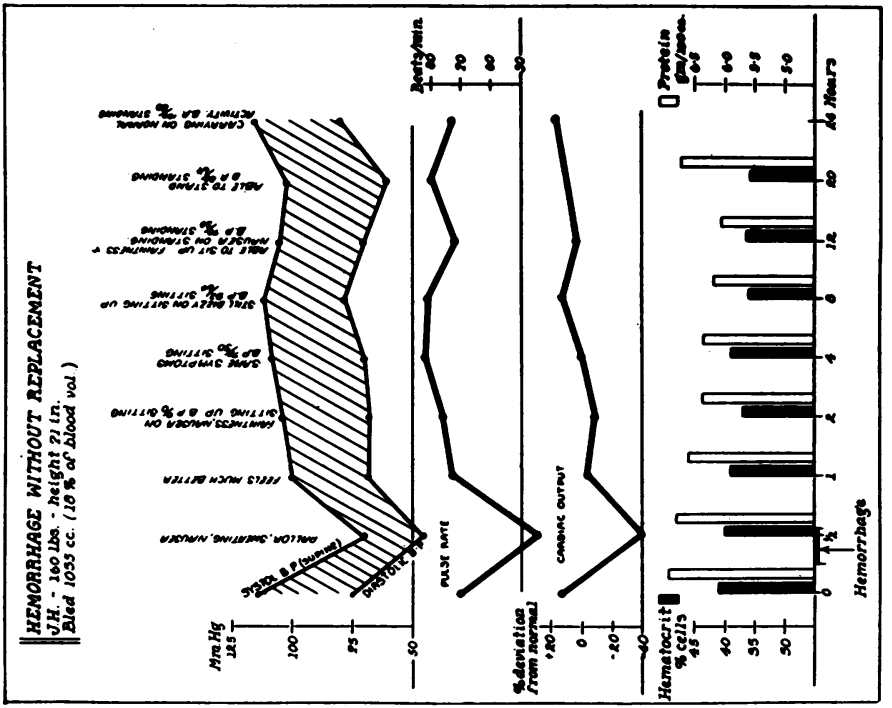

䆓

秃

i.

它

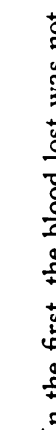


thirsty. The other patients, convalescent and taking fluids ad $l i b$, were judged to be normally hydrated. The results are recorded in Table V. They show that in the 4 patients who were well supplied with fluid the estimated increment of blood volume 3 hours after the end of the injection was larger, or but little less, than the amount injected. In the dehydrated patient, this increment 3 hours later was less than half the volume injected.

\section{DISCUSSION}

After the administration of gelatin solution intravenously, the plasma protein concentration and hematocrit diminish more than can be accounted for by the volume of fluid injected. This dilution slowly increases, reaching a maximum several hours after the injection. While some investigators ( 7 ) have believed that changes in plasma volume could be estimated from the hematocrit with reasonable accuracy, others $(24,25)$ have produced evidence that the errors in such an estimate were often great and that a similar estimate from changes in plasma protein concentration was also unsatisfactory. The frequent lack of concordance. between the results of estimates of blood volume made under similar conditions by methods based on different physiological principles makes the accuracy of any method difficult to judge, but the effect of gelatin on blood volume seems fairly clear. In dogs, given far larger amounts of gelatin in relation to blood volume than those we employed, it was found (26) that blood volume, as estimated by $\mathrm{T} 1824$, was markedly increased after the infusion, although the increment was generally somewhat less than the volume of the injected solution. In man, using the same dye, other authors (27) estimated that the increase of plasma volume, immediately after the injection of a gelatin, somewhat different from that we employed, averaged 58 per cent of the amount injected; but, thereafter, the blood volume continued to increase, the average maximal increment, 70 per cent of the amount injected, being found 4 hours afterwards. Results obtained in the Harrison Department of Research Surgery suggest that the small molecular gelatin used by these investigators (27) appears in the urine more rapidly than that employed in this
TABLE $V$

Plasma volumes calculated from dilution of $T 1824$ before and 3 hours after the intravenous injection of $500 \mathrm{ml}$. of 6 per cent gelatin solution

\begin{tabular}{|c|c|c|c|c|c|c|}
\hline \multirow{2}{*}{ Patient } & \multirow{2}{*}{ Weight } & \multicolumn{2}{|c|}{ Before injection } & \multicolumn{2}{|c|}{ After injection } & \multirow{2}{*}{$\begin{array}{l}\text { Change } \\
\text { in } \\
\text { plasma } \\
\text { volume }\end{array}$} \\
\hline & & $\begin{array}{l}\text { Plasma } \\
\text { volume }\end{array}$ & $\underset{\text { tocrit }}{\text { Hema- }}$ & $\begin{array}{l}\text { Plasma } \\
\text { volume }\end{array}$ & $\underset{\text { tocrit }}{\text { Hema- }}$ & \\
\hline $\begin{array}{l}\text { P. O. } \\
\text { S. E. } \\
\text { J. O. } \\
\text { E. M. } \\
\text { L. A. }\end{array}$ & $\begin{array}{c}\text { kgm. } \\
43 \\
55 \\
64 \\
60 \\
64\end{array}$ & $\begin{array}{c}m l . \\
1988 \\
2280 \\
2920 \\
2638 \\
2638\end{array}$ & $\begin{array}{c}\text { per cent } \\
42.5 \\
44.8 \\
44.3 \\
48.4 \\
52.4\end{array}$ & $\begin{array}{c}m l . \\
2785 \\
2710 \\
3160 \\
3040 \\
3590\end{array}$ & $\begin{array}{c}\text { per cent } \\
29.1 \\
39.3 \\
42.5 \\
40.8 \\
45.1\end{array}$ & $\begin{array}{l}m l . \\
695 \\
430 \\
240 \\
402 \\
952\end{array}$ \\
\hline
\end{tabular}

study. Nevertheless, our results are in essential accord with theirs, the dilution reaching its maximum in the samples taken 3 or 4 hours after injection, at which time the hematocrit and plasma protein values could be accounted for by assuming a degree of dilution approximately equal to the volume of fluid injected. The average increase in blood volume, as measured by $\mathrm{T} 1824$, is of this magnitude (Table V). Gelatin must be slowly lost from the blood stream, for it appears in the urine (1), but it also has the power of attracting fluid into the blood by its osmotic properties. Apparently, for the first few hours after injection, the second effect often predominates, and the volume added by injection may be maintained or even increased.

The results indicate that there is a small increase in venous pressure after gelatin infusion. Approximately equivalent increments were found by others (23) after infusion of similar amounts of serum.

The cardiac output increased significantly after the gelatin infusion in 3 of the 5 normal subjects (Table II), and this increase was much more conspicuous in all the 6 chronically ill patients (Table III). Even larger percentage increases of cardiac output were found (26) to follow gelatin infusion in dogs, but the amount of solution injected was much larger in proportion to the blood volume than was used in this study, so these results are concordant with ours.

In the case of the 3 subjects who were bled, one treated with gelatin, one with saline, and the other left untreated as a control, we were repeating on man a type of experiment performed in animals in many laboratories for the benefit of 
generations of medical students. In our subjects, blood pressure was diminished by the hemorrhage; it recovered only slowly without treatment, improved temporarily after saline, and more permanently after infusion of the colloid. Results such as these have been duplicated time and again in animal experiments. Much more conspicuous, however, were the differences obvious in our 3 subjects when they arose and attempted to stand. T. N., who had received gelatin, stood without a symptom at a time when A. F., who had received saline, stood only with some difficulty from dizziness; and J. H., who had received no replacement therapy, could not stand at all without fainting.

In general, our results with gelatin are in marked contrast to those observed after intravenous injections of electrolyte solutions ( 6 to 11). Relatively slow infusions of moderate volumes of gelatin produced marked circulatory changes which reached a maximum several hours after the completion of the infusion. Gelatin, leaving the circulation only slowly and drawing additional fluid into the blood, may increase the blood volume by an amount greater than the volume injected. While the plethora imposed no excessive strain on the cardiovascular system of our subjects, the results we obtained on chronically ill patients suggest the necessity for a good deal of caution in administering gelatin or other hyperosmotic macromolecular colloids to patients with diminished cardiac reserve. In fact, the precipitation of symptoms suggesting acute decompensation following the infusion of gelatin to such a patient has been reported (2).

The results of the hemorrhage and replacement experiments indicate clearly that gelatin is an effective agent in the restoration and maintenance of blood volume following acute hemorrhage, and that the symptoms of a hemorrhage as large as $15.0 \mathrm{ml}$. per $\mathrm{kgm}$. of body weight disappear after its use.

\section{SUMMARY}

1. Observations on pulse rate, blood pressure, venous pressure, cardiac output, plasma protein, and hematocrit have been made during and after intravenous infusions of 6 per cent ossein gelatin in normal subjects, chronically ill patients, and volunteers subjected to acute hemorrhage of 16 to 20 per cent of their blood volume. Estimates of the effect of such infusions on blood volume were made in 5 cases.

2. Gelatin infusion produced marked and sustained hemodilution in all of these cases. The calculated increases in blood volume were at times in excess of the actual volume of gelatin injected, indicating that the hyperosmotic gelatin solution draws additional extravascular fluid into the circulation. Emphasis is placed on the danger of overloading the circulation of certain patients.

3. Though pulse rate and blood pressure changed but little, cardiac output usually increased markedly after gelatin infusion. The maximum increase was generally found several hours after the end of the injection when blood dilution was at a maximum. Difficulties in the estimation of venous pressure after infusion of viscous solutions are discussed. There was usually a small increase of venous pressure after gelatin infusion.

4. Results obtained on volunteers subjected to large hemorrhage indicate that gelatin administered intravenously is an effective agent in restoring and maintaining the blood volume and in abolishing the symptoms which follow such acute hemorrhage.

\section{BIBLIOGRAPHY}

1. Parkins, W. M., Koop, C. E., Riegel, C., Vars, H. M., and Lockwood, J. S., Gelatin as a plasma substitute: with particular reference to experimental hemorrhage and burn shock. Ann. Surg., 1943, 118, 193.

2. Koop, C. E., Fletcher, A. G., Riegel, C., and Lockwood, J. S., Gelatin as a plasma substitute: a preliminary report of clinical experience. Surgery, 1944, 15, 839.

3. Koop, C. E., Fletcher, A. G., Riegel, C., Ratcliffe, H., and Parkins, W. M., Unpublished data.

4. Brunschwig, A., Corbin, N., and Johnston, C. D., Intravenous gelatin. Ann. Surg., 1943, 118, 1058.

5. Brunschwig, A., Scott, V. B., Corbin, N., and Moe, R., Observations on intravenous injection of gelatin for nutritional purposes. Proc. Soc. Exper. Biol. and Med., 1943, 52, 46.

6. Altschule, M. D., and Gilligan, D. R., Effects on cardiovascular system of fluids administered intravenously in man: dynamics of circulation. J. Clin. Invest., 1938, 17, 401.

7. Gilligan, D. R., Altschule, M. D., and Volk, M. C., Effects on cardiovascular system of fluids administered intravenously in man: studies of amount and 
duration of changes in blood volume. J. Clin. Invest., 1938, $17,7$.

8. Caughey, J. L., Jr., Effect of rapid infusion on venous pressure: test of cardiac reserve. Proc. Soc. Exper. Biol. and Med., 1934-35, 32, 973.

9. Richards, D. W., Jr., Caughey, J. L., Cournand, A., and Chamberlain, F. L., Intravenous saline infusion as clinical test for right-heart and left-heart failure. Tr. A. Am. Physicians, 1937, 52, 250.

10. Murphy, F. D., Correll, H., and Grill, J. C., Effects of intravenous solutions on patients with and without cardiovascular defects. J. A. M. A., 1941, 116, 104.

11. Hardy, J. C., and Godfrey, L., Unpublished data.

12. Robertson, J. D., Blood volume changes in health and hemorrhage and after various infusions. Lancet, $1938,2,634$.

13. Sharpey-Schafer, E. P., and Wallace, J., Circulatory overloading following rapid intravenous injections. Brit. M. J., 1942, 2, 304.

14. Meek, W. J., and Eyster, J. A. E., The effect of plethora and variations in venous pressure on diastolic size and output of the heart. Am. J. Physiol., 1922, 61, 186.

15. Eyster, J. A. E., and Middleton, W. S., Cardiovascular reactions to hemorrhage and transfusion in man. Am. J. Physiol., 1924, 68, 581.

16. Shenkin, H. A., Cheney, R. H., Govons, S. R., Hardy, J. D., Fletcher, A. G., and Starr, I., On the diagnosis of hemorrhage in man; a study of volunteers bled large amounts. Am. J. M. Sc., 1944, 208, 421.

17. Shenkin, H. A., Cheney, R. H., Govons, S. R., and Starr, I., Effects of acute hemorrhage of known amount on the circulation of essentially normal persons. Am. J. M. Sc., 1943, 206, 806.

18. Starr, I., and Schroeder, H. A., Ballistocardiogram: normal standards, abnormalities commonly found in diseases of heart and circulation, and their significance. J. Clin. Invest., 1940, 19, 437.
19. Gibson, J. G., 2nd, and Evans, W. A., Jr., Clinical studies of blood volume: relation of plasma and total blood volume to venous pressure, blood velocity rate, physical measurements, age and sex in ninety normal humans. J. Clin. Invest., 1937, 16, 317.

20. Moritz, F., and von Tabora, D., Ueber eine Methode, Beim Menschen den Druck in Oberflächlichen Venen Exakt zu Bestimmen. Deutsches Arch. f. klin. Med., Leipz., 1910, 98, 475.

21. Starr, I., Clinical studies on incoordination of the circulation as determined by the response to arising. J. Clin. Invest., 1943, 22, 813.

22. Ebert, R. V., Stead, E. A., Jr., and Gibson, J. G., Jr., Response of normal subjects to acute blood loss with special reference to mechanism of restoration of blood volume. Arch. Int. Med., 1941, 68, 578.

23. Wallace, J., and Sharpey-Schaffer, E. P., Blood changes following controlled hemorrhage in man. Lancet, 1941, 2, 393.

24. Hopper, J., Jr., Tabor, H., and Winkler, A. W., Simultaneous measurements of the blood volume in man and dog by means of Evans blue dye, T1824, and by means of carbon monoxide: I. Normal subjects. J. Clin. Invest., 1944, 23, 628.

25. Hopper, J., Jr., Winkler, A. W., and Elkinton, J. R., Simultaneous measurements of the blood volume in man and dog by means of Evans blue dye, T1824, and by means of carbon monoxide: II. Under abnormal conditions including secondary shock. J. Clin. Invest., 1944, 23, 636.

26. Holt, J. P., and Knoefel, P. K., Changes in plasma volume and cardiac output following the intravenous injection of gelatin, serum, and physiological solution. J. Clin. Invest., 1944, 23, 657.

27. Jacobson, S. D., and Smyth, C. J., Gelatin as substitute for plasma. Observations on its administration to human beings. . Arch. Int. Med., 1944, 74, 254. 